\title{
Simultaneous multiple allelic replacement in the malaria parasite enables dissection of PKG function
}

\author{
Konstantinos Koussis ${ }^{1}$ (1), Chrislaine Withers-Martinez ${ }^{1}$, David A Baker ${ }^{2}$, Michael J Blackman ${ }^{1,2}$ (1)
}

\begin{abstract}
Over recent years, a plethora of new genetic tools has transformed conditional engineering of the malaria parasite genome, allowing functional dissection of essential genes in the asexual and sexual blood stages that cause pathology or are required for disease transmission, respectively. Important challenges remain, including the desirability to complement conditional mutants with a correctly regulated second gene copy to confirm that observed phenotypes are due solely to loss of gene function and to analyse structure-function relationships. To meet this challenge, here we combine the dimerisable Cre (DiCre) system with the use of multiple lox sites to simultaneously generate multiple recombination events of the same gene. We focused on the Plasmodium falciparum cGMP-dependent protein kinase (PKG), creating in parallel conditional disruption of the gene plus up to two allelic replacements. We use the approach to demonstrate that PKG has no scaffolding or adaptor role in intraerythrocytic development, acting solely at merozoite egress. We also show that a phosphorylation-deficient PKG is functionally incompetent. Our method provides valuable new tools for analysis of gene function in the malaria parasite.
\end{abstract}

DOI 10.26508/lsa.201900626 | Received 9 December 2019 | Revised 6 March 2020 | Accepted 9 March 2020 | Published online 16 March 2020

\section{Introduction}

From the early documentation of targeted gene disruption in yeast by homologous recombination (1) to the use of site-specific recombinases (2) and the development of gene-editing tools such as CRISPR $(3,4)$, the ability to modify DNA has revolutionised understanding of gene function in model organisms and pathogens. Plasmodium spp., the protozoan parasites that are the aetiological agents of malaria, are responsible for more than 400,000 deaths per year (5), with Plasmodium falciparum causing the deadliest form of the disease. Widespread resistance to frontline antimalarial drugs and the absence of an effective vaccine make the identification of new antimalarial drug targets a necessity (6), but to achieve this, an improved understanding of the biology of the parasite is required. Transient transfection of Plasmodium was first reported almost 3 decades ago (7), but in part due to the haploid genome of the parasite, functional studies of essential genes in the asexual blood stages that are responsible for all the clinical manifestations of the disease have been extremely difficult.

Conditional deletion or rearrangement of DNA segments through activation of site-specific recombinases such as Cre has been the gold-standard system for gene editing in many model organisms, but attempts to adapt the Cre-lox system to blood stages of $P$. falciparum initially failed because of difficulties in suppressing constitutive activity of the recombinase $(8,9)$. This problem was solved with the adaptation of the dimerisable Cre (DiCre) system initially for Toxoplasma and subsequently for P. falciparum blood stages $(10,11)$. In this approach, Cre is expressed in the form of two enzymatically inactive domains, each of which is fused to a small rapamycin-binding protein. In the presence of rapamycin (RAP), the two fusion proteins heterodimerise, rapidly inducing Cre activity (12, $13,14,15)$. The versatility of this system in Plasmodium was substantially enhanced with the development of the loxpint module, which elegantly allowed intragenic introduction of "silent" loxP sites within a small synthetic intron (16). Over the past $5 \mathrm{yr}$, additional modifications have been made to the P. falciparum Dicre system, including installation of the DiCre cassette into alternative chromosomal loci and use of different $P$. falciparum strains, and the approach has now been exploited for the functional analysis of many essential genes $(17,18,19,20,21,22,23,24,25,26,27,28,29)$. Very recently, DiCre has been adapted to other Plasmodium species, including the widely-used rodent malaria model Plasmodium berghei and the zoonotic pathogen Plasmodium knowlesi $(30,31)$. However, application of the system for the simultaneous generation of both loss-of-function and genetically complemented parasite lines has remained technically challenging.

Signalling through cyclic 3',5'-guanosine monophosphate (cGMP) plays important roles in many eukaryotes, including the malaria parasite. The only known sensor of CGMP signalling in the parasite is its CGMP-dependent protein kinase (PKG), which is encoded by a single-copy gene in all Plasmodium species $(32,33)$. Chemical genetic and genetic approaches have shown that cGMP

${ }^{1}$ Malaria Biochemistry Laboratory, Francis Crick Institute, London, UK ${ }^{2}$ Faculty of Infectious and Tropical Diseases, London School of Hygiene \& Tropical Medicine, London, UK 
signalling is essential for key developmental transitions throughout the entire parasite life cycle, including activation of sexual forms (gametogenesis), ookinete formation and motility in the mosquito vector, sporozoite motility and liver cell infection, and maturation and release (egress) of liver-stage merozoites into the bloodstream of the vertebrate host $(34,35,36,37,38,39,40)$. In the asexual blood stages of $P$. falciparum, the use of highly selective small-molecule inhibitors of PKG, combined with the use of gatekeeper mutagenesis to confirm on-target selectivity of the compounds, has convincingly demonstrated that PKG catalytic activity is not required during the $\sim 48$-h-long period of intraerythrocytic parasite development (schizogony) but has an essential role in asexual blood-stage schizont rupture and merozoite egress (37, 38, 41). More recently, conditional down-regulation of PKG expression using a destabilisation domain approach confirmed that the kinase is required for blood-stage viability; however, in this study, the specific point(s) in the erythrocytic cycle at which proliferation arrested was not documented (42). Many kinases and other signalling proteins operate within multicomponent signalling complexes in which individual members play critical scaffolding or adaptor roles in addition to any canonical enzymatic roles (43, $44,45,46,47)$. The various studies alluded to above have clearly indicated that PKG enzyme activity is not required for intraerythrocytic parasite development. However, whether PKG plays additional scaffolding role(s) throughout intraerythrocytic development that may not require catalytic activity is unknown. Many other aspects of PKG biology are also still unexplored, including the importance of PKG phosphorylation, a modification that often plays a role in kinase activation and function (48). Several global phosphoproteomic studies have identified a total of seven residues that are phosphorylated in PKG; two of these lie within a cyclic nucleotide-binding domain and five within the kinase domain (49, $50,51,52,53)$. Whether phosphorylation of these residues is important for PKG function has not been examined.
Here, we address these important questions. To enable this, we describe a modified DiCre system that takes advantage of stochastic Cre-mediated recombination between different lox sites (54), allowing us to perform in parallel conditional disruption and allelic replacement of the $P$. falciparum PKG gene, with the distinct events indicated by the expression of distinct fluorescent reporter proteins. Our new system provides valuable new tools for conditional genetics in Plasmodium. Using it, we genetically demonstrate for the first time that PKG has no essential scaffolding role during intraerythrocytic malaria parasite development, and we show that a phosphorylation-deficient form of PKG is nonfunctional.

\section{Results}

\section{Introduction of two contiguous lox sites into a short intron to allow multiple simultaneous conditional gene modifications}

We first re-designed the loxpint module (16) to incorporate two contiguous non-overlapping 34-bp sequences, loxN and lox2272. These modified lox sites have previously been shown to be incompatible with loxP or with each other in different systems $(54,55)$. To validate the functionality of the modified loxpint (called 2loxPint) and to assess its capacity to undergo correct splicing, we inserted the 2loxPint element into the $P$. falciparum gene encoding the cGMP-dependent PKG. The P. falciparum PKG gene (pfpkg: PF3D7_1436600) comprises 4 introns and 5 exons, with all four of its consensus cyclic nucleotide-binding domains and the kinase domain encoded by exons 3-5. Using a Cas9 expression plasmid (19) to mediate targeted double-stranded DNA cleavage and a marker-free rescue construct to enable repair by homologous recombination, we precisely replaced intron 3 of pfpkg with 2loxpint in the Dicreexpressing $P$. falciparum B11 line (Fig $1 \mathrm{~A}$ and B) (25). Limiting dilution cloning of the modified parasites generated a genetically homogenous
A

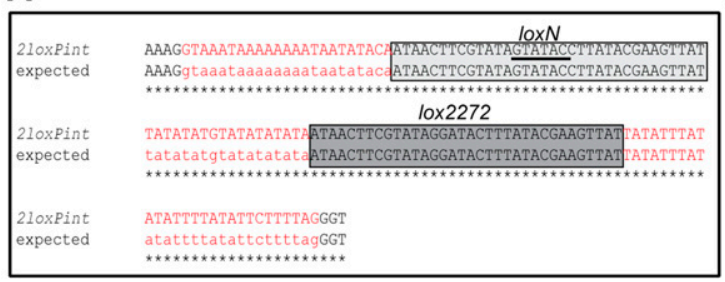

C

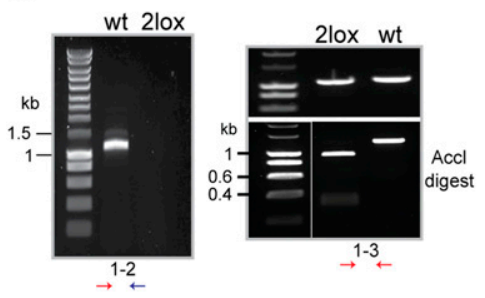

D

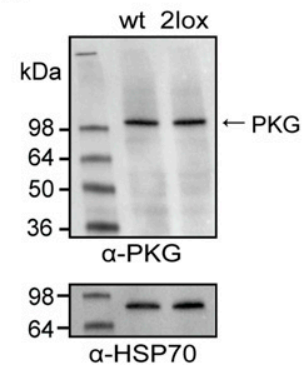

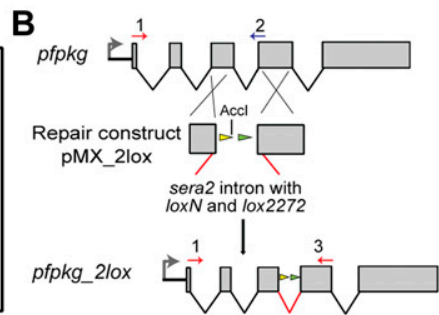

E

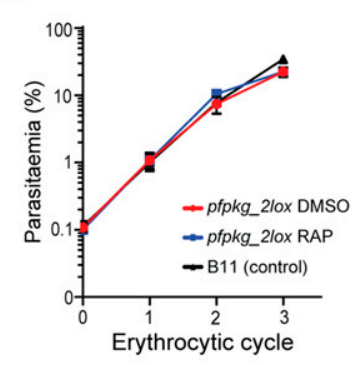

Figure 1. Replacement of pfpkg intron 3 with 2loxPint allows normal PKG expression and parasite replication.

(A) Shown is the integrated 2loxPint sequence derived by nucleotide sequencing of genomic DNA from the P. falciparum pfpkg_2lox line, aligned with the expected sequence (both in red). The loxN and lox2272 sites are in grey boxes, with the unique internal Accl site underlined. Boundaries of the $3^{\prime}$ end of exon 3 (AAAG) and the $5^{\prime}$ end of exon 4 (GGT) are shown in black. (B) Schematic representation of the strategy used to replace intron 3 of the pfpkg gene with 2loxPint in the DiCre-expressing B11 P. falciparum line. Positions of oligonucleotides used for diagnostic PCR are indicated (red and blue arrows). (C) (Left) Diagnostic PCR results showing absence of the endogenous intron 3 in the pfpkg_2lox line (2lox) relative to the parental $\mathrm{B} 11$ line (wt). (Right) Diagnostic PCR results and restriction digest of the PCR amplicon with AcCl, showing the expected digestion only of the amplicon from the pfpkg_2lox line (2lox). (D) Western blot analysis of extracts of wt and pfpkg_2lox schizonts, showing similar expression levels of PKG ( 98 kD, indicated). Antibodies to the cytoplasmic parasite protein HSP70 were used as a loading control. (E) Growth curves showing replication of DMSO-treated (control) or RAP-treated pfpkg_2lox parasites relative to the parental line B11. Mean values are shown from triplicate experiments. Error bars \pm SD $(n=3)$. 
parasite line called pfpkg_2lox. Correct integration of the 2loxPint intron into the pfpkg locus of these parasites was verified by diagnostic PCR and confirmed by restriction digest analysis and Sanger sequencing of the PCR amplicon (Fig 1B and C). Western blot analysis with a polyclonal anti-PKG antibody confirmed that levels of PKG expression in pfpkg_2lox schizonts were indistinguishable from those of the parental B11 line (Fig 1D). To assess whether the 2loxpint modification could affect parasite fitness or lead to undesirable gene disruption by DiCre-mediated recombination within the intron (which was not expected given the incompatibility of the loxN and lox2272 sites), replication of the pfpkg_2lox parasites was monitored after treatment with RAP or vehicle only (DMSO control). This confirmed in both cases normal growth rates relative to the parental B11 line (Fig 1E). Collectively, these results showed that replacement of the endogenous pfpkg intron 3 with 2loxpint produced no detectable defect in PKG expression or parasite growth, in turn indicating efficient splicing of the 2loxpint intron.

\section{An allelic replacement approach for simultaneous disruption and complementation of the pfpkg gene}

Having established the pfpkg_2lox parasite line, we next further genetically modified the parasites to enable simultaneous disruption and replacement of the pfpkg gene, using the paired, mutually incompatible lox sites within the 2loxpint intron. To do this, we once again used a marker-free Cas9-mediated strategy to adapt the $3^{\prime}$ flanking sequence of the pfpkg locus (Fig 2A). Additional loxN and lox2272 sites were introduced, positioned within intervening sequences such that Dicre-mediated recombination between the two loxN sites (one within the 2loxpint and one downstream of the pfpkg ORF) was expected to reconstitute sequence encoding a full-length PKG fused to the fluorescent protein eGFP. In contrast, recombination between the two lox2272 sites would instead severely truncate the endogenous PKG gene, simultaneously fusing the residual $\mathrm{N}$-terminal 92 residues of the protein to mCherry. We reasoned that use of the two fluorescent reporter proteins in this way would facilitate microscopic detection of the expected recombination events after induction of Dicre activity $(35,56)$.

Correct integration of the $3^{\prime}$ targeting construct was confirmed by PCR (Fig S1A) and a clonal parasite line was obtained by limiting dilution. As expected, transient RAP treatment of this line, termed pkg:WGFP-CkOR, resulted in the generation of eGFP and mCherryexpressing parasites, corresponding to recombination between either the two loxN sites, leading to a perfect allelic replacement (PKGsynth_GFP), or between the two lox2272 sites, resulting in disruption of the pfpkg gene and expression of mcherry ( $\triangle P K G$ m $m$ Cherry). The expected recombination events were detectable by diagnostic PCR (Fig 2B), and expression of the predicted fusion proteins in RAP-treated parasite cultures was confirmed by Western blot using antibodies to GFP and mCherry (Fig 2C). Quantitation by flow cytometry and live microscopy of the eGFP and mCherry-positive parasites at the end of the erythrocytic cycle of RAP treatment (henceforth referred to as cycle 0) indicated that these were formed at a ratio of $\sim 9: 1$ (Fig 2D), suggesting that recombination between the lox $\mathrm{N}$ sites was substantially favoured, probably as a result of the relatively short distance between these sites in the modified pkg:WGFP-CkoR genome (Fig 2A). Collectively, these results confirmed that simultaneous generation of two genetically distinct populations within a single erythrocytic cycle after RAP treatment is achievable in $P$. falciparum.

\section{PKG has no scaffolding role during intraerythrocytic parasite development}

Previous chemical genetic studies on asexual blood-stage $P$. falciparum have shown that selective inhibition of PKG activity prevents discharge of specialised secretory organelles called micronemes and exonemes, with a resulting block in egress (38). However, whether PKG expression plays a non-enzymatic role throughout the erythrocytic life cycle has not been addressed genetically.

The generation in a single step of readily distinguishable populations of PKGsynth_GFP (expected to be phenotypically wild type) and $\triangle \mathrm{PKG}$ _mCherry (PKG-null) parasites allowed us to now examine the effects of PKG disruption or reconstitution on the entire asexual blood-stage life cycle under identical conditions. To do this, we first examined replication of control (DMSO-treated) and RAP-treated pkg:WGFP-CkOR parasites over the course of three erythrocytic cycles ( $72 \mathrm{~h}$ ). Because of the high proportion of PKGsynth_GFP parasites in the RAP-treated cultures, we expected to see only very small differences in overall replication rates in the cultures over this period, a prediction that was confirmed experimentally (Fig 3A). However, examination of the RAP-treated cultures revealed complete loss of the mCherry-expressing $\triangle \mathrm{PKG}$ _mcherry parasites by the end of cycle 1, suggesting a severe growth defect upon PKG disruption (Fig 3B). To analyse this defect in more detail over the course of a single erythrocytic cycle, a highly synchronous culture containing ring-stage pkg:WGFP-CkOR parasites (parasitaemia $\sim 6.5 \%$ ) was RAP-treated and then incubated for a further $40 \mathrm{~h}$ to allow development to mature schizont stage, the point in the lifecycle at which PKG expression peaks (57). Microscopic examination over the course of this period revealed the appearance of both eGFP-expressing and mCherry-expressing mature schizonts as expected, with no decrease in total parasitaemia relative to that at the start of that cycle. This showed that all or most RAP-treated parasites were able to mature normally over the course of cycle 0 , in turn indicating that ablation of PKG expression does not affect subsequent intraerythrocytic maturation within that cycle. Samples of the culture were then assessed by flow cytometry at intervals over the ensuing $3 \mathrm{~h}$. As shown in Fig 3C, this revealed a gradual time-dependent decrease in the proportion of PKGsynth_GFP schizonts, presumably due to rupture of these parasites as they reached full maturity and underwent merozoite egress. In contrast, the total proportion of $\triangle P K G_{-}$mCherry parasites gradually increased over the 3-h period, suggesting a selective defect in rupture as mature schizonts accumulated. To further quantify this under conditions where the schizonts made up a greater proportion of the total cell population, we enriched mature cycle 0 schizonts from similar cultures and again used flow cytometry to selectively examine time-dependent changes in the proportions of fluorescent cells in the enriched schizont population. This revealed a greater than twofold increase over a 3 -h period in the proportion of 
A

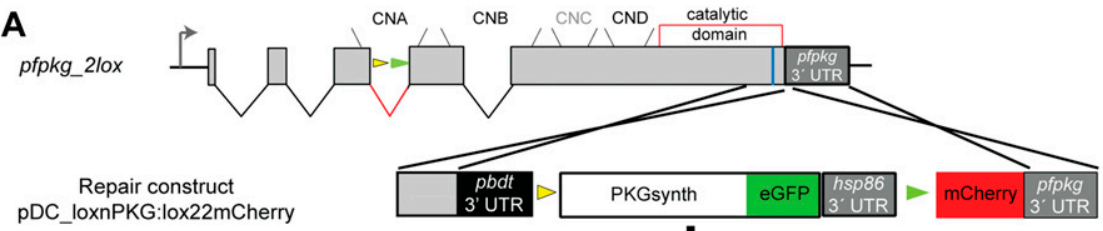

recombination event 1

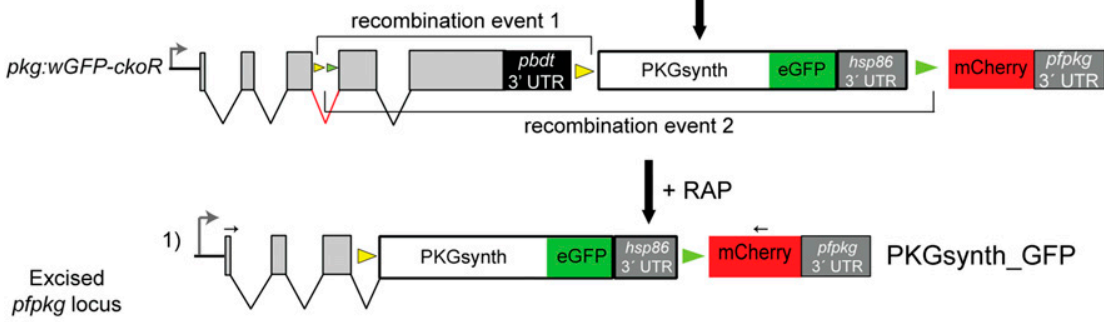

2)

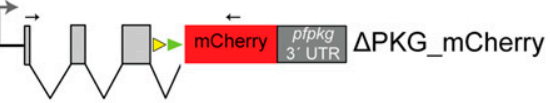

B

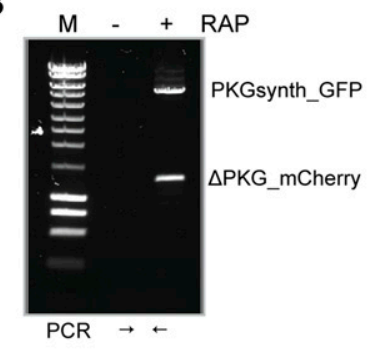

C

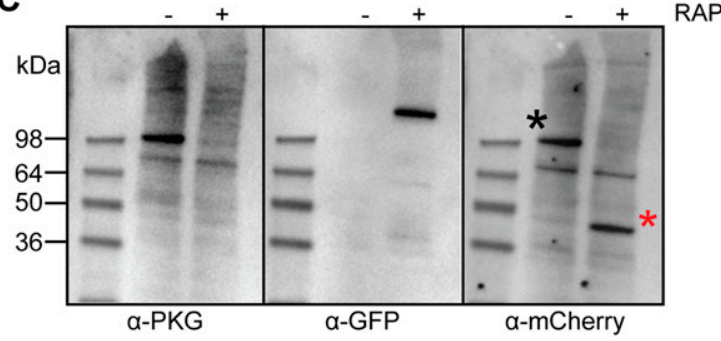

D

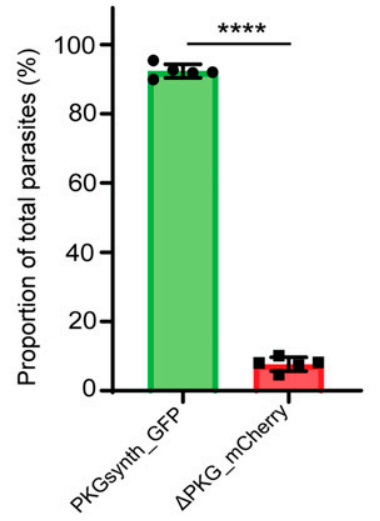

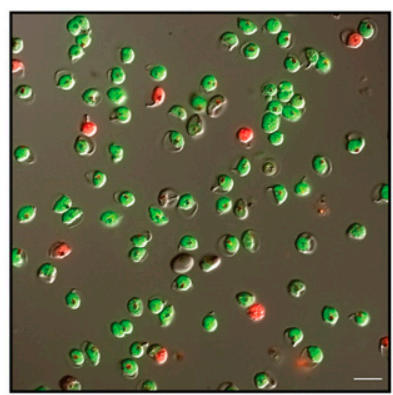
RAP

\section{(a)} corresponding to both predicted recombination events upon amplification from genomic DNA of RAPtreated pkg:WGFP-ckoR parasites. Expected sizes of the amplicons corresponding to PKGsynth_GFP and $\triangle P K G \_m C h e r r y$ are 5.6 and $1.3 \mathrm{~kb}$, respectively. Multiple attempts to amplify the corresponding region from mock-treated parasites (-RAP) failed, probably because of the large size of the predicted amplicon $(\sim 10 \mathrm{~kb})$. (c) Western blot showing loss of expression of PKG upon RAP treatment of pkg:WGFP-ckoR parasites, concomitant with appearance of signals corresponding to the PKG_GFP fusion (expected molecular mass $125 \mathrm{kD}$ ) and the mCherry fusion (expected mass $38 \mathrm{kD}$; red asterisk). After being probed with rabbit anti-PKG antibodies (left-hand blot), the membrane was re-probed with a rabbit anti-mcherry antibody to demonstrate the appearance of mcherry only in the RAP-treated sample and to highlight the difference between the DMSO- and the RAP-treated samples for both PKG (black asterisk) and mCherry. Note that the PKG_GFP fusion is not recognised by the commercially available PKG antibody because of masking of the C-terminal epitope, as previously observed (57). (D) (Left) Quantification by flow cytometry of the relative proportions of PKGsynth_GFP and

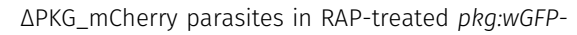
ckoR parasites (sampled at the end of cycle 0 ). Individual values (dark circles or squares) are shown from five biological replicate experiments $(n=5)$, and mean values are indicated as bars. Statistical significance was determined by unpaired $t$ test $(P$-value $<0.0001)$. (Right) Representative image from differential inference contrast/fluorescence microscopic examination of RAP-treated pkg:wGFP-ckoR parasites (end of cycle 0), showing both GPF- and mCherry-positive schizonts. Scale bar, $10 \mu \mathrm{m}$.

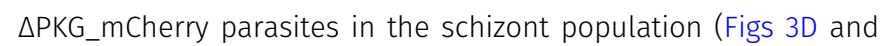
S2), whereas the proportion of PKGsynth_GFP schizonts again decreased. This further supports a selective arrest in egress in the $\triangle \mathrm{PKG}$ _mCherry mutants.

To finally examine the phenotype of PKG disruption more closely, we used time-lapse video microscopy to visualise parasite fate at egress. For this, mature schizonts enriched from RAP-treated pkg: $W G F P$-ckoR cultures were further incubated for $3 \mathrm{~h}$ in the presence of the reversible PKG inhibitor 4-[7-[(dimethylamino)methyl]-2-(4fluorphenyl)imidazo[1,2- $\alpha$ ]pyridine-3-yl]pyrimidin-2-amine (compound 2). This prevents egress while allowing schizonts to reach full maturation, effectively synchronising the schizonts at a state of high maturation. Removal of compound 2 from wild-type parasites leads to PKG activation and schizont rupture within minutes, which can be monitored microscopically. As shown in Fig 3E and Video 1, this showed that only the PKGsynth_GFP parasites underwent egress, whereas the $\triangle \mathrm{PKG}$ _mCherry parasites displayed no signs of parasitophorous vacuole rupture or any of the other morphological changes that generally precede egress $(26,58,59,60)$, remaining trapped inside their host red blood cells. Taken together, these results convincingly demonstrate that PKG has no detectable noncatalytic scaffolding role during maturation of asexual blood-stage 
A

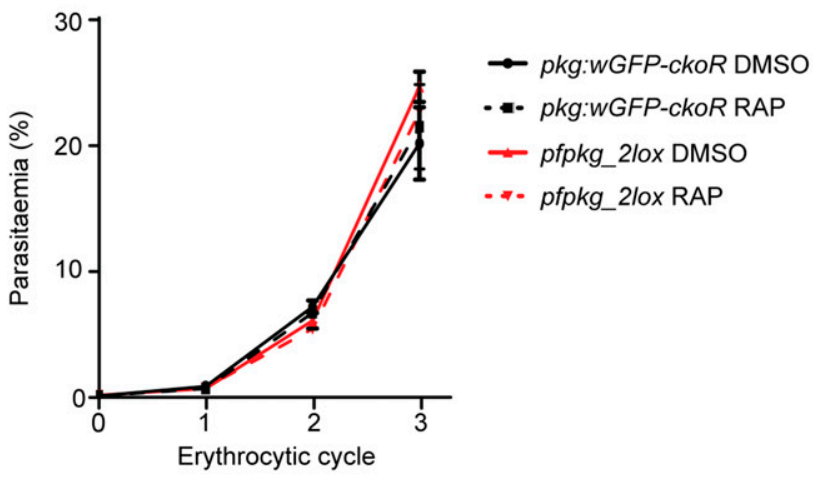

B

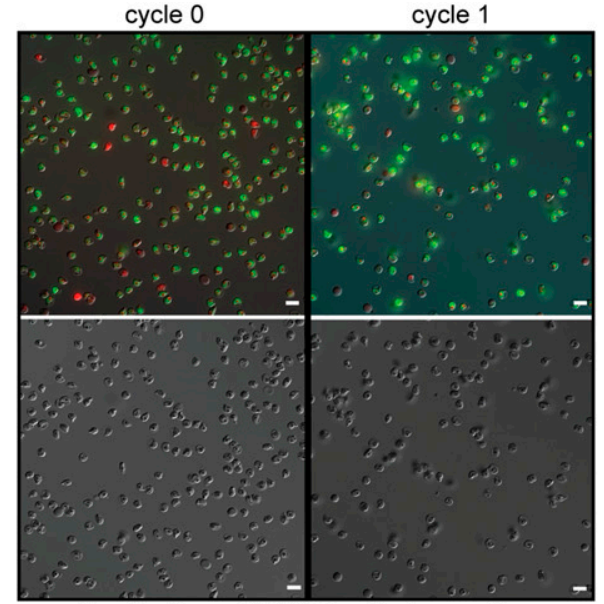

Replication of RAP treated parasites

C

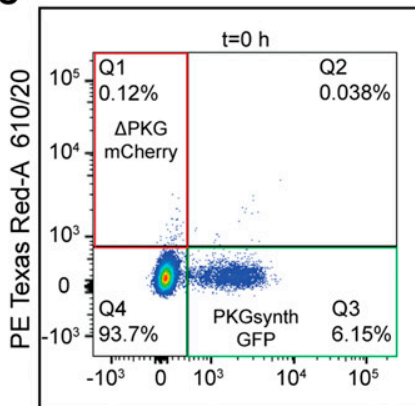

Time course of egress
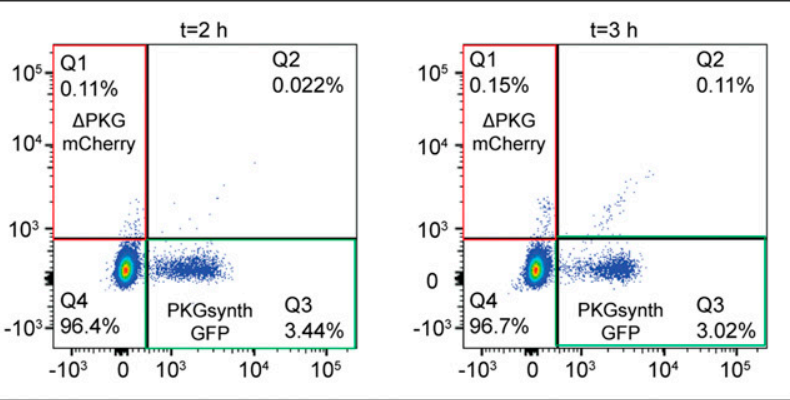

FITC-A 530/30

D

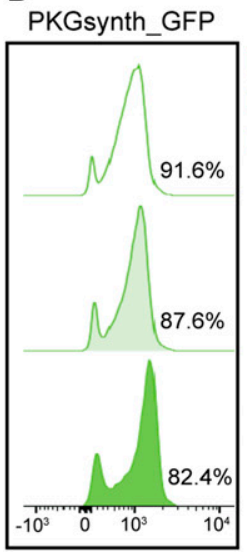

E

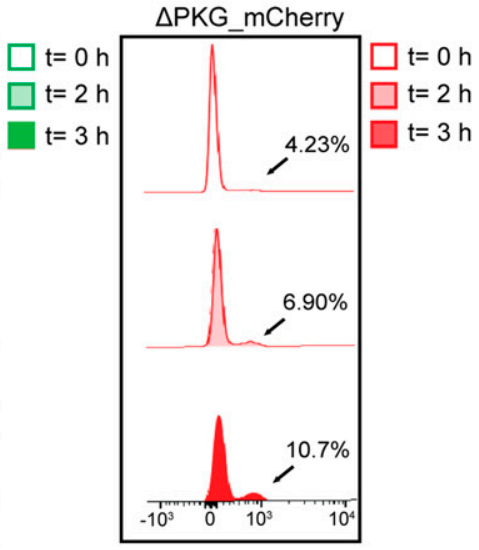

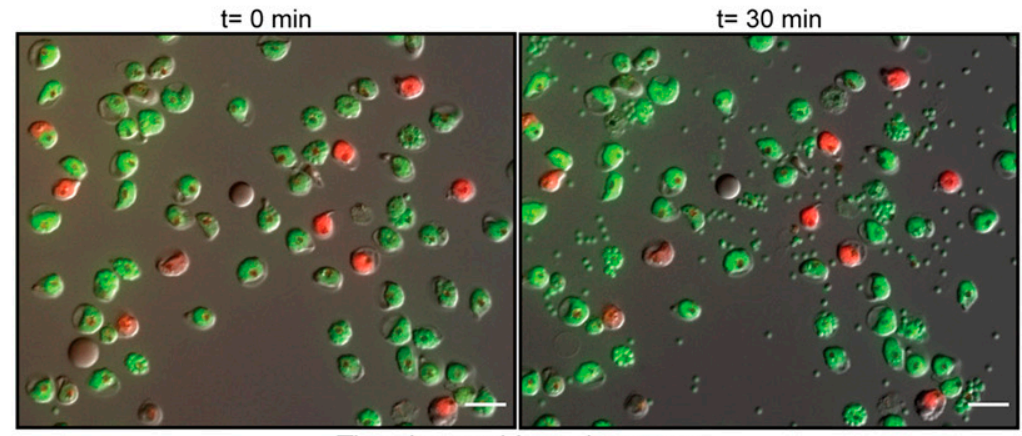

Time-lapse video microscopy

Figure 3. PKG-null parasites undergo normal intraerythrocytic development but arrested egress.

(A) Growth curves showing replication of DMSO-treated (control) or RAP-treated pkg:wGFP-ckOR and pfpkg_2lox parasites. Percentage parasitaemia values are shown (quantified by flow cytometry). Error bars $\pm S D(n=3)$. (B) Differential inference contrast/fluorescence images of schizonts from a RAP-treated pkg:WGFP-ckoR culture, showing virtual disappearance of $\triangle P K P_{-} m$ Cherry parasites by the end of cycle 1. (C) Two-parameter dot plot representation of flow cytometry data monitoring the relative proportions of PKGsynth_GFP schizonts (green box, Q3; lower right-hand quadrant) and $\triangle$ PKG_mCherry schizonts (red box, Q1; upper left-hand quadrant) with time. Monitoring was initiated $\sim 4 \mathrm{~h}$ after RAP treatment of a highly synchronous pkg:wGFP-ckoRc culture. The percentage of each population at each time point is shown within the relevant quadrant. The Q4 population predominantly represents uninfected erythrocytes. Parasitaemia at the point of RAP-treatment (the start of cycle 0) was 6.5\%. (D) Histogram depiction of flow cytometry analysis of schizonts enriched $\sim 44 \mathrm{~h}$ after RAP treatment of a pkg:wGFP-ckoRc culture, showing time-dependent accumulation of

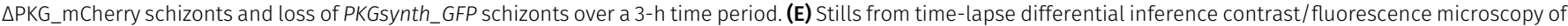
isolated, RAP-treated pkg:wGFP-ckoR schizonts after release of a compound 2-mediated egress block, showing that only the PKGsynth_GFP schizonts undergo rupture and merozoite egress. No rupture of the $\triangle \mathrm{PKG} \_\mathrm{mCherry}$ schizonts was observed even after prolonged imaging. Scale bars, $10 \mu \mathrm{m}$. 
schizonts, while confirming that it is essential for schizont rupture and merozoite egress.

\section{Removal of phosphosites renders PKG nonfunctional}

Previous studies of the Cre-lox system in other organisms have demonstrated that the efficiency of Cre-mediated site-specific recombination generally decreases as a function of the linear "distance" between chromosomal loxP sites (61). In the pkg:wGFPckoR line, the genetic distance between the loxN and lox2272 recombination events was 3.6 and $7.7 \mathrm{~kb}$, respectively. We reasoned that this more than twofold difference was likely responsible for the relatively inefficient generation of the $\triangle \mathrm{PKG}$ _mCherry parasites (the lox2272 recombination event; Fig 2A). To examine whether we could obtain more similar ratios of the two recombination events, facilitating comparative analysis of the resulting genetically distinct populations, we designed a modified strategy in which recombination between the more closely situated lox sites would result in gene disruption, whereas recombination between the pair of more spatially distant lox sites would lead to allelic replacement.

To test and validate this system, we focused on the importance of the known phosphorylation sites within P. falciparum PKG. A total of seven phosphosites (Fig 4A) have previously been identified through several independent phosphoproteome studies by different groups $(49,50,51,52,53)$. To examine the essentiality of phosphorylation at these sites, we decided to compare the phenotype resulting from PKG disruption with that resulting from allelic replacement with a mutant form of PKG in which the seven Ser, Thr, or Tyr residues that are targets of phosphorylation were replaced with Ala residues.

Repair construct pDC_loxNmCherry:lox22PKG (Fig S1B) was introduced into pfpkg_2lox parasites and, after limiting the dilution cloning, correct modification by homologous recombination of the modified pfpkg locus was confirmed by PCR. RAP treatment of the resulting parasite line, called pkg:CkoR-mutGFP, resulted in the expected recombination events as determined by diagnostic PCR (Fig $4 \mathrm{~B}$ and $\mathrm{C}$ ). As predicted given the more similar distances between the compatible lox sites in this parasite line, ratios of GFP and mcherry-positive schizonts at the end of cycle 0 were roughly comparable, demonstrating efficient generation of both $\triangle$ PKG_mCherry and PKGmut_GFP transgenic parasites in the same culture after a single RAP treatment (Fig 4D). Also as expected, the mature schizonts that appeared at the end of cycle 0 in the RAPtreated cultures were morphologically indistinguishable from those in control, DMSO-treated cultures, further indicating normal intracellular development in all the fluorescent parasites despite the fact that approximately half of the RAP-treated culture lacked expression of full-length PKG (Fig 4E), whereas the other half expressed the PKGmut_GFP mutant in which all seven phosphorylated residues were substituted with Ala residues.

To investigate the long-term viability of these parasites, RAPtreated or DMSO-treated pkg:CkoR-mutGFP cultures were monitored in parallel for three erythrocytic cycles. A complete arrest of parasite proliferation was observed in the RAP-treated cultures (Fig 4F). To compare the egress phenotype of the PKGmut_GFP parasites with that of the $\triangle P K G$ _mCherry parasites, we again used time-lapse microscopy to monitor egress, using as controls schizonts of the parental pkg:ckoR-mutGFP line. As shown in Fig $4 \mathrm{G}$ and Video 2, the PKGmut_GFP schizonts displayed an egress defect identical to that of the $\triangle P K G$ m $m$ Cherry parasites. To corroborate these findings, we monitored the appearance in culture supernatants of SERA5, an abundant parasitophorous vacuole protein which is released into culture supernatants upon egress $(38,62)$. As shown in Fig $4 \mathrm{H}$, processed SERA5 was completely absent from culture supernatants of the RAP-treated pkg:ckoR-mutGFP schizonts. These experiments clearly showed that Ala substitution of its seven known phosphosites renders PKG functionally inactive, producing a phenotype that mimics conditional disruption of the pfpkg gene.

\section{Expanding the DiCre toolkit with the introduction of the 3loxPint module}

To unambiguously rule out a non-catalytic role for PKG during intraerythrocytic parasite growth and to expand the utility of our new toolbox for simultaneous creation of multiple allelic replacements, we examined whether it was possible to introduce a third different lox sequence into the loxpint intron to allow a third potential outcome after induction of DiCre activity. For this, we added a lox71 site to the 2loxPint, creating module 3loxPint (Fig S3A and $\mathrm{B}$ ). Cre-mediated recombination between lox71 and the lox66 site creates a unique lox72-mutant site, which is also incompatible with $\operatorname{lox} P(25,63)$. As previously, we first precisely replaced the endogenous intron 3 of pfpkg in the P. falciparum B11 line with the 3loxpint module. A clonal modified parasite line was obtained (called pfpkg_3lox) and the modification verified by diagnostic PCR, restriction digest analysis, and nucleotide sequencing (Fig S3B and C). Western blot analysis of pfpkg_3lox schizonts with PKG-specific antibodies showed no differences in PKG expression levels between this line, pfpkg_2lox and parental B11 parasites (Fig S3D), indicating correct splicing of the 3loxpint.

To enable detection of individual recombination events, we decided as previously to design downstream modifications of the modified pfpkg_3lox gene such that each distinct recombination event would lead to expression of a different fluorescent protein. To validate the system and to further examine the effects of pfpkg disruption, we decided to design the system such that all three recombination events would lead to conditional truncation of pfpkg (Fig 5A), enabling us to follow maturation of these parasites under conditions in which essentially the entire culture comprised PKG-null parasites. To do this, pfpkg_3lox schizonts were transfected with repair construct plasmid pDC_3cKO and clonal line pkg: 3cko obtained (Fig S1C). Synchronous ring-stage cultures of pkg: $3 c k o$ were then treated with RAP and the resulting cycle 0 schizontstage parasites were examined by diagnostic PCR using distinct primers designed to detect each predicted potential outcome of recombination between the various lox sites. All three expected recombination events were confirmed (Figs 5B and S4), supported by fluorescence microscopy examination which showed the presence of schizonts expressing mTagBFP2, eGFP, or mCherry (Fig 5C). Differential counts showed that these parasites were present in the population at proportions of $11.8 \% \pm 1.98 \%, 81.6 \% \pm 3.2 \%$, and $6.6 \% \pm 1.2 \%$, respectively $(n=2)$, indicating a strong preference for recombination between the loxN sites. This latter result also confirmed correct splicing of all versions of the modified intron 
A

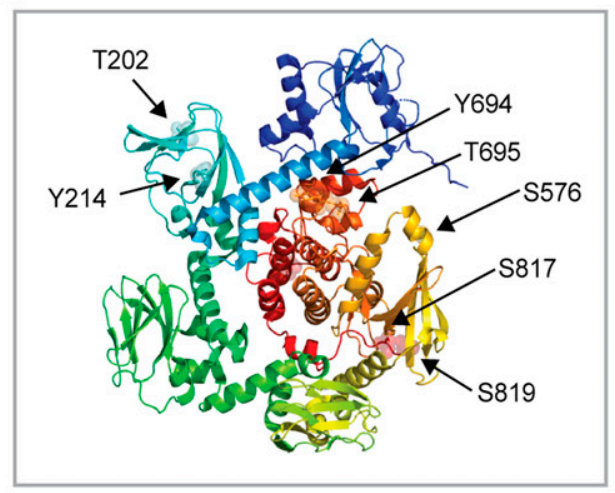

B

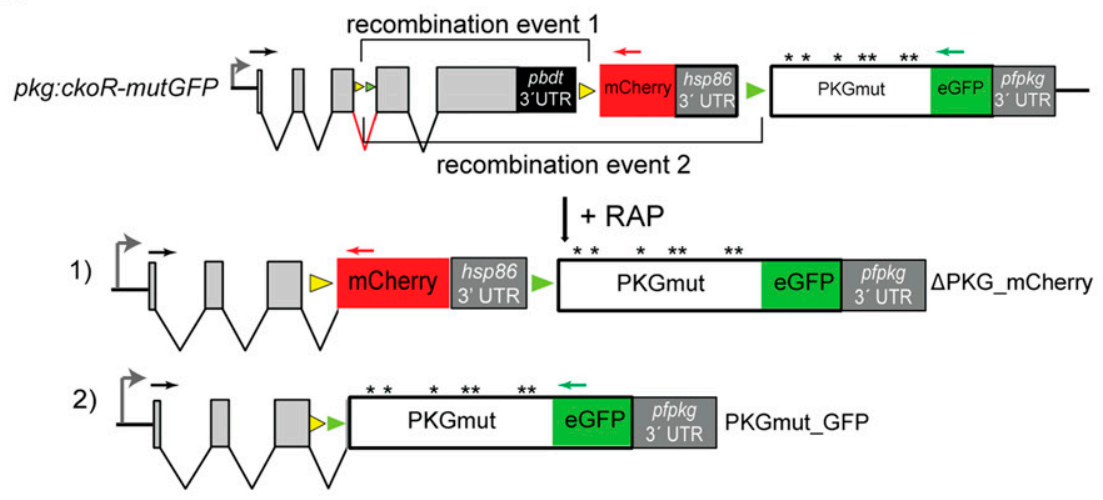

E

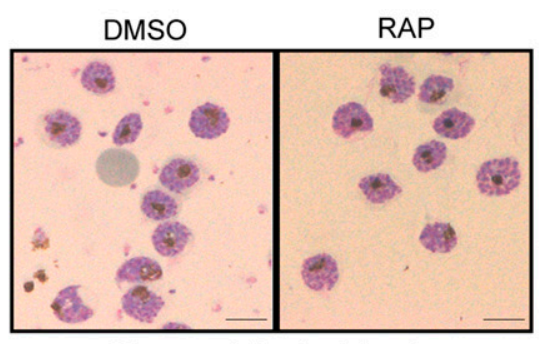

Giemsa-stained schizonts
$\mathbf{F}$

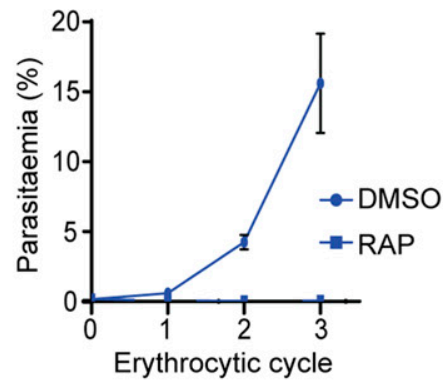

C

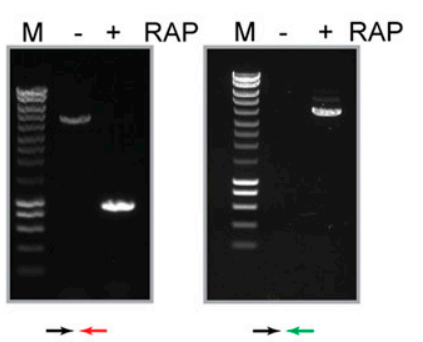

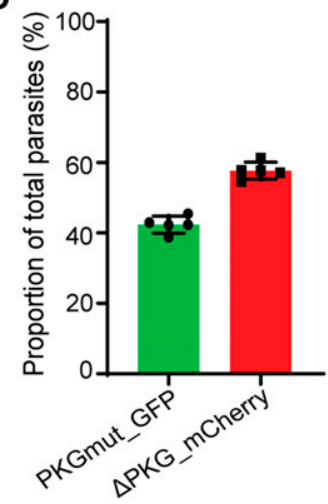

G

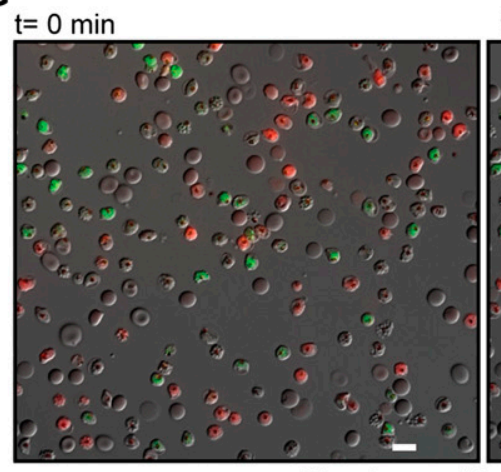

$t=30 \mathrm{~min}$

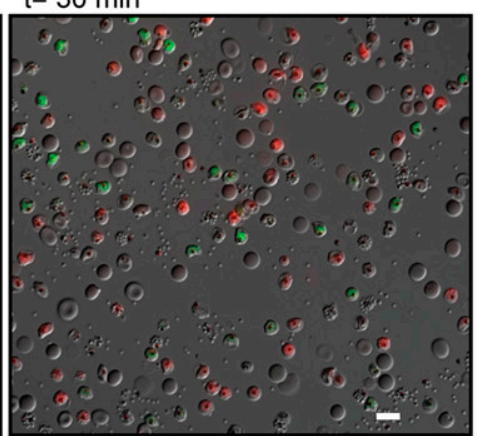

H

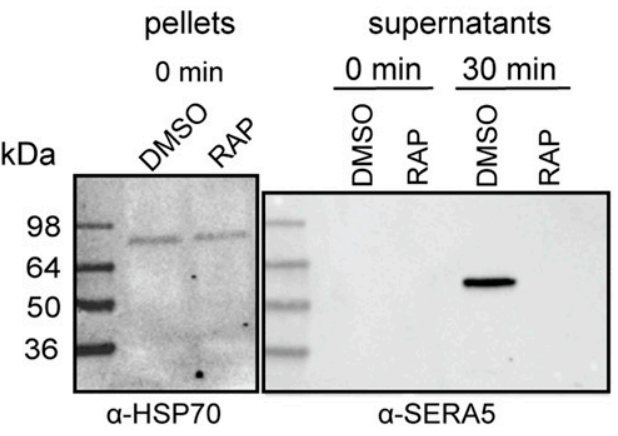

Time-lapse video microscopy

Figure 4. Phosphosite mutations render $P$. falciparum PKG inactive.

(A) Cartoon of the P. falciparum PKG x-ray crystal structure (PDB ID: 5DYK) in its apo form with rainbow colouring (N terminus in dark blue; C terminus in red). Cyclic nucleotide-binding domains A (dark blue), B (cyan), C (green), and D (lime) are shown, whereas the central kinase domains are in yellow/orange/red. Phosphosites identified by mass spectrometry are indicated and shown as sticks within colour-matching transparent spheres. The image was ray-traced in the PyMOL Open-Source Molecular Graphic System (https://pymol.org/2/). (B) Schematic of the modified pfpkg locus in the pkg:cKOR-mutGFP parasite line. Upon DiCre induction with RAP, recombination event 1 leads to conditional gene disruption ( $\triangle \mathrm{PKG}$-mCherry), whilst recombination event 2 leads to replacement of the endogenous allele with a partially synthetic full-length allele containing Ala substitutions of all seven phosphosites (asterisks), fused to GFP (recombination event 2; PKGmut_GFP). (C) Diagnostic PCR results showing detection of the two distinct recombination events after DiCre activation. The amplicon specific for $\triangle$ PKG_mCherry (denoted by the black and red arrows) is $\sim 1$ $\mathrm{kb}$ in the RAP-treated sample and $\sim 4.9 \mathrm{~kb}$ in the mock-treated (non-excised) sample. The amplicon specific for PKGmut_GFP is $\sim 4 \mathrm{~kb}$ in the RAP-treated sample. Amplification from mock-treated samples was unsuccessful, likely because of the large size of the predicted fragment. (D) Quantification of the ratio between PKGmut_GFP and $\triangle$ PKG_mCherry schizonts in the RAP-treated parasite population at the end of cycle 0 . Data shown are from five independent experiments; individual and mean values are shown. Error bars $\pm S D(n=5)$. $(E)$ Giemsa-stained images of Percoll-enriched schizonts isolated at the end of cycle 0 of DMSO- and RAP-treated pkg:CKORmutGFP parasites, showing no discernible morphological differences. Scale bar, $10 \mu \mathrm{M}$. (F) Replication of DMSO- and RAP- treated pkg:ckoR_mutGFP parasites over three erythrocytic cycles. Parasitaemia values shown (obtained by flow cytometry) are averages of three independent experiments. Error bars \pm SD $(n=3)$. (G) Both PKGmut_GFP and $\triangle$ PKG_mCherry schizonts are defective in egress. Still images of the first and final frame of a 30-min time-lapse video of mock-treated (grey) or RAPtreated (green and red) pkg:ckoR_mutGFP parasites. Schizonts were synchronised by incubation with the reversible PKG inhibitor compound 2, then washed, mixed in equal proportions, and monitored for egress over a period of $30 \mathrm{~min}$. Neither the red ( $\triangle \mathrm{PKG}$ _mCherry) nor the green (PKGmut_GFP) schizonts underwent egress, whereas most of the parental mock-treated pkg:ckoR_mutGFP schizonts ruptured. Scale bar, $10 \mu \mathrm{M}$. (H) Western blot showing that no SERA5 P50 was released into culture supernatants of RAP-treated pkg:ckoR_mutGFP schizonts, consistent with impaired egress in both the PKGmut_GFP and $\triangle$ PKG_mCherry schizonts. 
A
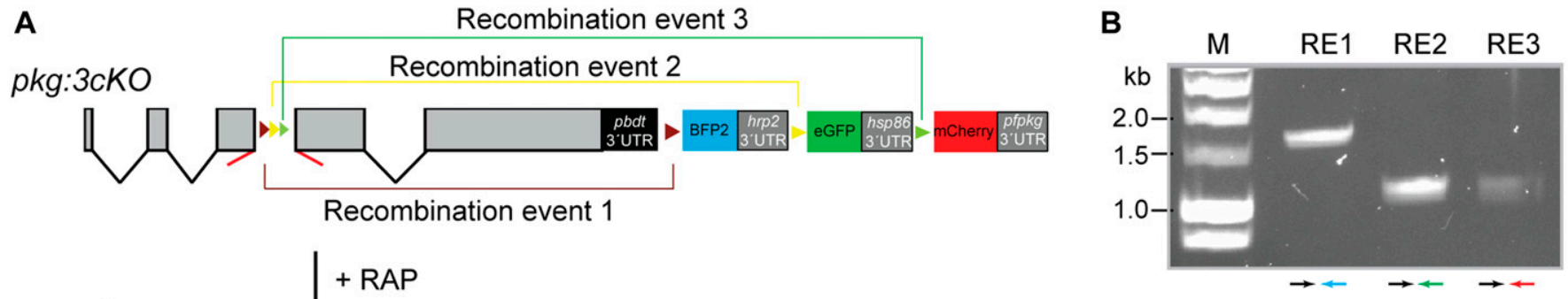

1)

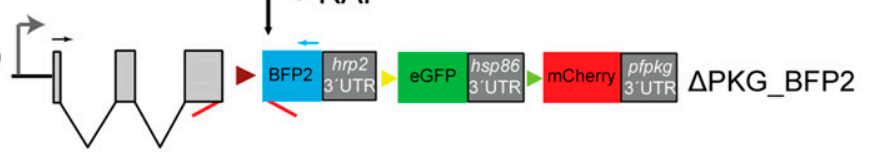

2) $\vec{\square}$

3) $\vec{\perp}$
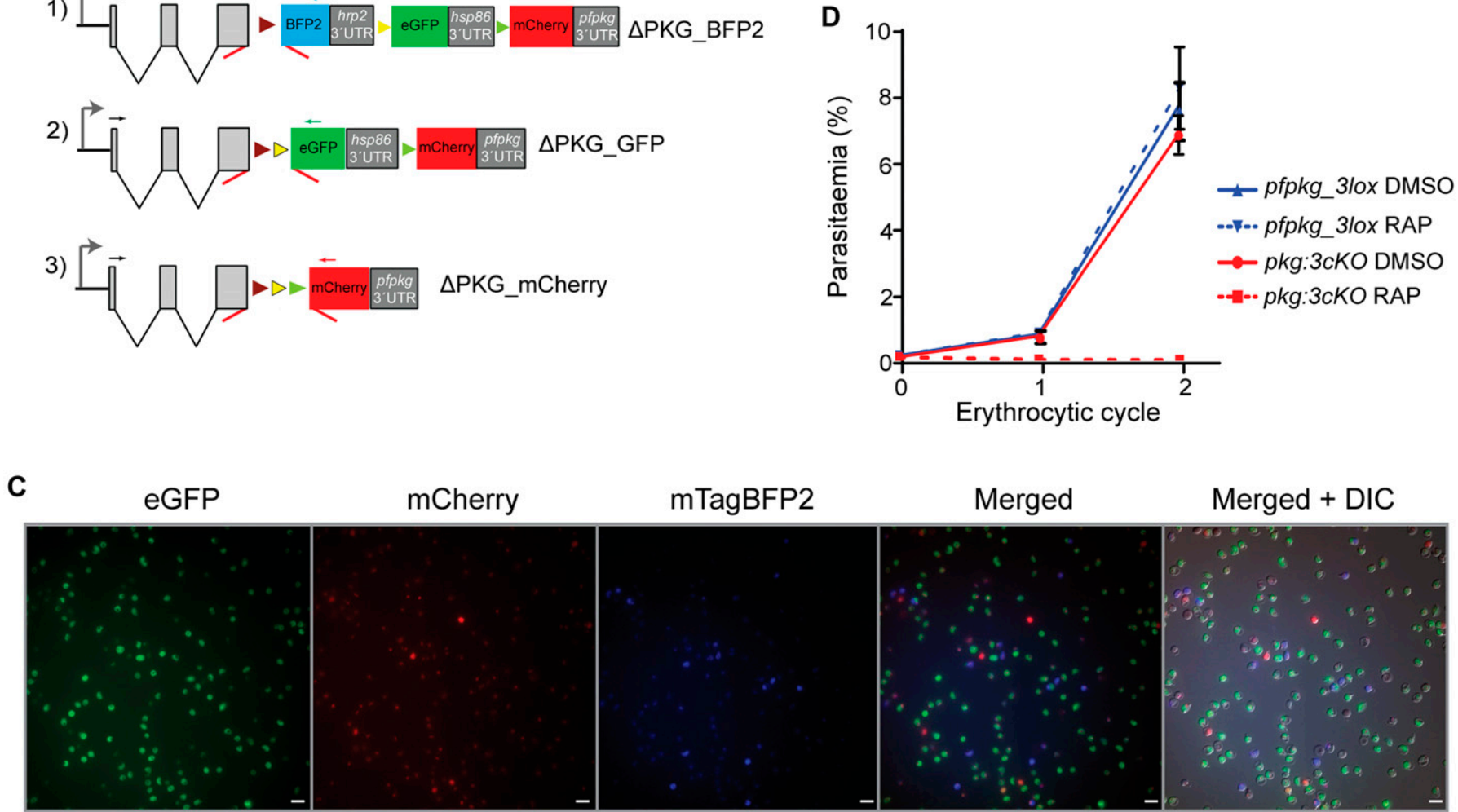

Figure 5. Simultaneous generation of three distinct allelic exchange events.

(A) Schematic of the approach used to conditionally disrupt pfpkg and create three distinct knockout parasite populations expressing either mTagBFP2 ( $\triangle$ PKG_BFP2),

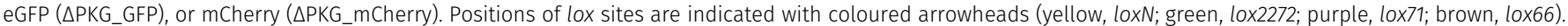
Positions of oligonucleotide primers used for diagnostic PCR are indicated (coloured arrows). (B) Confirmation by diagnostic PCR of the three recombination events (RE1, RE2, and RE3, respectively) in RAP-treated pkg:3cKO parasites. Coloured arrows represent identify of the primers used. (C) Growth assay of the pfpkg_3lox and pkg:3cKO parasite lines after mock-treatment (DMSO) or treatment with RAP. Parasitaemia was measured by flow cytometry. Error bars \pm SD $(n=6)$. (D) Fluorescent microcopy images of live RAP-treated pkg:3cKO schizonts from the end of cycle 0, confirming the presence of all three fluorescent populations. Scale bars, $10 \mu \mathrm{M}$.

after recombination. Importantly, there was no reduction in parasitaemia over the course of cycle 0 despite the high levels of fluorescent parasites at schizont stage, proving that disruption of PKG had no effect on intraerythrocytic parasite development. Comparison of pfpkg_3lox and pkg:3cKO parasite replication over ensuing cycles showed that RAP treatment of pkg:3cKO parasites led to a complete arrest in parasite growth (Fig 5D), as expected.

\section{Discussion}

The use of site-specific recombinases and mutually incompatible lox sites has been a powerful tool for conditional mutagenesis and gene expression in many organisms, with a notable example being multicolor labelling of tissues for the study of neuronal and developmental circuits in metazoa $(54,64,65,66)$. Here, we have effectively adapted this principle for use in the malaria parasite to address key challenges in Plasmodium genetics and to gain crucial new insights into the role of PKG in parasite blood-stage development. By introducing two or three distinct lox sites into an artificial Plasmodium intron we have been able for the first time to analyse simultaneously up to three distinct allelic replacements of PKG, allowing us to demonstrate that PKG has no essential scaffolding role during intraerythrocytic development and to genetically confirm its essentiality for egress. This is despite the fact that PKG has been shown to be expressed in asexual stages as early as $24 \mathrm{~h}$ post invasion, with levels of expression reaching a maximum in late schizogony (57). 
Genetic complementation of Plasmodium knockouts with either a wild-type second copy of the gene to rescue the observed phenotype or a mutant copy to examine the role of specific residues or domains can be challenging. Episomal complementation, although technically simple and the method of choice in cases where multiple mutants need to be screened (e.g., (67)), can sometimes be partially successful because of poor segregation of plasmids in Plasmodium, leading to highly variable levels of expression in individual parasites and the need to maintain transgenic parasite populations under selective drug pressure $(68,69)$. Complementation by integration into a nonessential locus is preferable but again full restoration of the phenotype is not always possible because constitutive expression of a second gene copy can affect parasite fitness or correct trafficking of the gene product $(26,70,71$, 72). Complementation studies in animal models of malaria require extensive use of animals or the creation of mutants from independent transfections (73). The preferred method of choice is to insert a complementing allele into the authentic endogenous locus so that expression is driven by the native promoter, as described here with our new system.

The importance of genetic distance between cre-mediated recombination events has been previously documented in other systems (61) and was also evident in our data. Experiments in ES cells and mice have revealed three important parameters in site-specific recombination: a) levels of Cre expression (which in our study should be the same in all parasites); b) the genetic distance between lox sites; and c) the nature of the DNA sequence (74). In parallel, studies of two distinct mutants of a gene of interest, an unequal ratio of the different recombination events is therefore to be expected, especially in the case of larger genes. This could limit the utility of the 3loxpint system. In addition, our 3 loxPint results suggested a preference for recombination between lox $N$ sites as compared with recombination between lox2272 or lox71/lox66 pairs. Multiple site-specific recombination is to our knowledge, a largely unexplored area in Plasmodium. It would be interesting in future experiments to study the impact of different lox sites on recombination ratios.

The new loxpint modules described here represent useful additions to the expanding toolkit $(75,76,77,78,79)$ for conditional Plasmodium genetic modification, allowing up to three modifications of a gene of interest to be studied in parallel. The use of fluorescent markers combined with flow cytometry can also facilitate enrichment of the resulting parasite populations, which can be used subsequently for biochemical or phenotypical studies more readily than by generating and analysing independent lines. The system has allowed us to improve understanding of the role of PKG in blood-stage egress, providing an exciting background for further investigation of the function of this essential enzyme and its potential as a drug target.

\section{Materials and Methods}

\section{Reagents and antibodies}

The antifolate drug WR99210 was from Jacobus Pharmaceuticals. Rapamycin was from Sigma-Aldrich and used to treat parasites at 20 nM. The PKG inhibitor (4-[7-[(dimethylamino)methyl]-2-(4-fluorphenyl) imidazo[1,2- $\alpha$ ]pyridine-3-yl]pyrimidin-2-amine) (compound 2) was stored at $-20^{\circ} \mathrm{C}$ as a $10 \mathrm{mM}$ solution in DMSO and used in cultures at $1 \mu \mathrm{M}$. For PKG detection, a rabbit polyclonal human-PKG antibody (Enzo) was used at a dilution of 1:1,000. The GFP-specific mAb 11814460001 (Roche) was used at a dilution of 1:1,000, as was a polyclonal rabbit anti-mCherry (ab167453; Abcam). A polyclonal rabbit anti-SERA5 antibody was used at 1:2,000 (80). The anti-HSP70 antibody (used at 1:1,000) was a kind gift of Dr Ellen Knuepfer, Francis Crick Institute. Restriction enzymes were from New England BioLabs and DNA ligations were performed with the Rapid DNA ligation kit (Roche).

\section{P. falciparum culture, transfection, and synchronisation}

The B11 DiCre-expressing P. falciparum line (25) was maintained at $37^{\circ} \mathrm{C}$ in human erythrocytes in RPMI 1640 containing Albumax II (Thermo Fisher Scientific) supplemented with $2 \mathrm{mM}$ L-glutamine and was used for all genetic modifications described. Cultures were routinely microscopically examined using Giemsa-stained thin blood films and mature schizonts were isolated by centrifugation over 70\% (vol/vol) isotonic Percoll (GE Healthcare, Life Sciences) cushions. Highly synchronous ring-stage cultures were obtained by allowing schizonts to invade fresh erythrocytes for $1-2 \mathrm{~h}$ under shaking conditions followed by a second round of Percoll treatment and treatment of the final pellet with $5 \% \mathrm{D}$-sorbitol to lyse residual schizonts.

Transfections were performed as previously described (81). In brief, $10^{8}$ Percoll-enriched schizonts were resuspended in $100 \mu \mathrm{l}$ of P3 primary cell solution (Lonza) containing $20 \mu$ g of Cas9 expression plasmid and $60 \mu \mathrm{g}$ of linearised donor plasmid. Program FP158 of the Amaxa 4D Nucleofector X (Lonza) was used for electroporation. Drug selection with 2.5 nM WR99210 started $24 \mathrm{~h}$ post transfection for two cycles. Clonal lines were obtained by serial limiting dilution in flat-bottomed 96-well plates (82). Single plaques were selected and grown in the presence of $1 \mu \mathrm{M}$ 5-fluorocytosine (5-FC, provided as clinical grade Ancotyl) to select for Cas9 plasmid-free and marker-free parasites.

For parasite genomic DNA extraction, the QIAGEN DNeasy Blood and Tissue kit was used. Genotype analysis diagnostic PCR was performed using Phusion polymerase (New England Biolabs).

In all cases, DiCre activity was induced by transient RAP treatment of highly synchronous early ring-stage parasites (2-3 h post invasion) as previously described (10). Parasite samples for PCR analysis of DiCre-mediated excision were collected $24 \mathrm{~h}$ after initiation of RAP treatment. Samples for Western blot analysis were collected at $42 \mathrm{~h}$ post initiation of RAP treatment.

\section{Plasmid construction and genotyping of transgenic lines}

Sequences of the fragments used for all parasite modifications in this study are provided in Supplemental Data 1.

Parasite line pfpkg_2lox was created by replacing the third intron of pfpkg with 2loxPint (the P. falciparum 3D7 sera2 intron containing loxN and lox2272 sequences, respectively). A DNA fragment was commercially obtained comprising $447 \mathrm{bp}$ upstream of intron 3 as the 5' homology arm, the2loxPint module, and 436 bp downstream 
of intron 3 as the $3^{\prime}$ homology arm (GeneArt, Thermo Fisher Scientific). A single guide RNA targeting sequence TTTTAGGGTCATACTTTTT was inserted into a previously described pDC2 plasmid expressing Cas9, resulting in plasmid pDC2-2loxg (19). The repair plasmid (pMX_2lox) was linearised with BgIII overnight and transfected into parasites together with plasmid pDC2_2loxg. Integration was confirmed by PCR, using primers exon1_For and exon4_Rev and restriction digest of the PCR amplicon with Accl (a full list of oligonucleotide primers used in this study is provided in Table S1). Absence of the endogenous intron 3 was confirmed by using primers exon1_For and intron3_Rev.

Construct pDC_loxnPKG:lox22mCherry was used to create line pkg:WGFP-CkOR. and was based on vector PDC_mCherry_MCS (26). The construct contains in tandem 1) a 5' homology arm of 399 bp endogenous and $321 \mathrm{bp}$ synthetic pfpkg sequence (obtained as a gBlock from IDT) with the PbDT 3' UTR, 2) a fragment comprising loxN, the 3' 46 bp of the sera2 intron, a synthetic fragment of pfpkg starting from exon 4 (the synthetic pfpkg cDNA cloned in vector pTrcHis was used as a template) fused to the eGFP coding sequence (56) and the pfhsp86 3' UTR, and 3) the lox2272 sequence the 3' 46 bp of the sera2 intron, the mCherry coding sequence and the pfpkg 3'UTR as the $3^{\prime}$ homology arm. A single guide RNA-targeting sequence TTGGCCGGTTAATATATCA was cloned into the Cas9 vector, generating plasmid pDC2_pkg. Vector pDC_loxnPKG:lox22mCherry was linearised overnight with Scal and transfected together with the pDC2-pkg Cas9 plasmid into P. falciparum line pfpkg_2lox. Correct $5^{\prime}$ and $3^{\prime}$ integration was verified by PCR using primers K2_For/5int_Rev and PKGsynth_For/3int_Rev, respectively. Absence of the endogenous locus was confirmed using primers exon1_For/PKGutr_Rev.

Line pkg:ckoR-mutGFP was generated using repair plasmid pDC_loxNmCherry:lox22PKG. The relevant Ala substitutions were made either by overlapping PCR (for substitutions of T202A, Y214A, and S576A) using the synthetic pfpkg as a template, or were obtained as a gBlock fragment (Y694A, T695A, S817A, and S819A) from IDT. To create the plasmid, a synthetic fragment containing the loxN sequence, the $3^{\prime} 46 \mathrm{bp}$ of the sera 2 intron, the mCherry gene, the pfhsp86 3' UTR followed by lox2272, the 3' 46 nucleotides of the sera2 intron and the first $100 \mathrm{bp}$ of a recodonised pfpkg exon 4 was commercially obtained (GeneArt, Thermo Fisher Scientific). The fragment was isolated by digest with $\mathrm{Hpal} / \mathrm{Ncol}$ and cloned into a plasmid containing the rest of the mutant synthetic pfpkg gene fused to eGFP, followed by the pfpkg 3' UTR. This intermediate vector was digested with $\mathrm{Hpal} / \mathrm{Hind} \mathrm{III}$ and the 5' homology arm used in construct pDC_loxnPKG:lox22mCherry was cloned in, resulting in the final vector. This was linearised with Scal overnight and transfected into line pfpkg_2lox with the pDC2-pkg Cas9 vector. Correct integration was confirmed as described above for line pkg: WGFP-ckoR.

For the pfpkg_3lox line, intron 3 of pfpkg was replaced with the 3 loxpint module (the sera2 intron containing lox71, loxN, and lox2272 sequences, respectively). A plasmid (pMX_3lox) containing the module spanned by the same $5^{\prime}$ and $3^{\prime}$ homology arms as used for line pfpkg_2lox was commercially obtained (GeneArt, Thermo Fisher Scientific). Plasmid pMX_3lox was linearised overnight with $\mathrm{Ncol}$ and transfected it together with plasmid pDC2_2loxg into $P$. falciparum B11 schizonts. Integration and loss of the endogenous pfpkg intron 3 was confirmed by PCR and restriction digest analysis of the amplicon with Accl.

Construct pDC_3cKO was used to create line pkg:3cKO. Initially a plasmid, pMX_3cKO-1, containing 1) the lox66 site followed by the 3' $46 \mathrm{bp}$ of the sera2 intron and the mTagBFP2 coding sequence with the pfhrp2 3' UTR and 2) an loxN site followed by the 3' 46 bp of the sera2 intron and the eGFP coding sequence was commercially obtained (GeneArt, Thermo Fisher Scientific). This plasmid was linearised by restriction digest with Bglll. A fragment containing the hsp86 3' UTR, the lox2272, the 3' 46 bp of the sera2 intron, mCherry and the pfpkg 3'UTR as the 3' homology arm was isolated from plasmid pDC_loxnPKG:lox22mCherry and cloned into vector pMX_3cKO-1, resulting in construct pDC_3cKOint. This intermediate plasmid was digested with $\mathrm{Hpal}$ and $\mathrm{Nhel}$ and a 3.2-kb fragment was isolated and cloned into vector pDC_loxnPKG:Lox22mCherry previously digested with the same enzymes, resulting in plasmid pDC_3cKO. This was linearised overnight with Scal and transfected together with the pDC2_pkg plasmid into P. falciparum line pfpkg_3lox.

\section{Parasite growth assays}

To determine parasite growth rates, synchronous ring-stage parasites at $0.1 \%$ parasitaemia and $2 \%$ haematocrit were dispensed in triplicate into 12-well plates. Samples of $50 \mu \mathrm{l}$ from each well were collected at 0, 2, 4, and 6 d, stained with SYBR Green, and analysed by flow cytometry on a BD FACSVerse using BD FACSuite software. Data were analysed using FlowJo software.

\section{Parasite egress assay}

Parasite culture supernatants were prepared as previously described (28). In brief, mature schizonts were isolated by Percoll centrifugation and incubated for a further $3 \mathrm{~h}$ in complete medium containing compound $2(1 \mu \mathrm{M})$. After removal of the inhibitor, schizonts were immediately resuspended in fresh serum-free RPMI at $37^{\circ} \mathrm{C}$ to allow egress. Schizont pellets and culture supernatants at $\mathrm{t}=0$ were collected as a control sample, whereas culture supernatants were collected by centrifugation after $30 \mathrm{~min}$.

\section{Flow cytometry analysis}

Parasites expressing eGFP or mCherry were quantified by flow cytometry using a FACS Aria flow cytometer (BD Biosciences). Samples were initially screened using forward and side scatter parameters and gated for erythrocytes. For eGFP detection, a 488 $\mathrm{nm}$ Blue Laser was used with a 530/30 filter, whereas for mCherry, a $561 \mathrm{~nm}$ Yellow-Green Laser was used with a 610/20 filter. For mTagBFP2 detection, the BD FACSVerse was used with a 450/50 filter.

\section{Immunoblotting}

Synchronised schizonts were isolated by Percoll gradient centrifugation and washed in RPMI 1640 without Albumax. Parasites were extracted into a Triton X-100 buffer (20 mM Tris- $\mathrm{HCl}, \mathrm{pH} 7.4,150 \mathrm{mM}$ $\mathrm{NaCl}, 0.5 \mathrm{mM}$ EDTA, and 1\% vol/vol Triton X-100, supplemented with 
$1 \times$ protease inhibitors [Roche]). Extracts were incubated on ice for $30 \mathrm{~min}$ then clarified by centrifugation at $12,000 \mathrm{~g}$ for $15 \mathrm{~min}$ at $4^{\circ} \mathrm{C}$. Supernatants were mixed with SDS sample buffer containing DTT and incubated for $5 \mathrm{~min}$ at $95^{\circ} \mathrm{C}$ before fractionation by SDS-PAGE analysis on 4-15\% Mini-PROTEAN TGX Stain-Free Protein Gels (BioRad). Transfer to nitrocellulose membranes and probing for Western blot analysis was as described previously (38).

\section{Time-lapse and live fluorescence microscopy}

Viewing chambers were constructed as previously described (38). Images were recorded on a Nikon Eclipse Ni light microscope fitted with a Hamamatsu C11440 digital camera and Nikon N Plan Apo $\lambda$ $63 \times / 1.45 \mathrm{NA}$ oil immersion objective. For time-lapse video microscopy, differential inference contrast images were taken at 10-s intervals over $30 \mathrm{~min}$, whereas fluorescence (GFP, mTagBFP2, and mCherry) images were taken every 2 min to prevent bleaching. Time-lapse videos were analysed and annotated using Fiji (83).

\section{Statistical analysis}

All statistical analysis was carried out using GraphPad Prism 8.

\section{Supplementary Information}

Supplementary Information is available at https://doi.org/10.26508/lsa. 201900626.

\section{Acknowledgements}

The authors are grateful to Ellen Knuepfer for the kind gift of the $\alpha$-HSP70 antibody and to Robert Moon for sharing unpublished information on the use of mTagBFP2 in Plasmodium. This work was supported by Wellcome Trust grant 106239/Z/14/A (K Koussis and MJ Blackman), Wellcome Trust grant $106240 / Z / 14 / Z$ (DA Baker), and Wellcome ISSF2 funding to the London School of Hygiene \& Tropical Medicine. The work was also supported by funding to MJ Blackman from the Francis Crick Institute (https:// www.crick.ac.uk/), which receives its core funding from Cancer Research UK (FC001043; https:/ / www.cancerresearchuk.org), the UK Medical Research Council (FC001043; https://www.mrc.ac.uk/), and the Wellcome Trust (FC001043; https://wellcome.ac.uk/). The funders had no role in study design, data collection and analysis, decision to publish, or preparation of the manuscript.

\section{Author Contributions}

K Koussis: conceptualization, data curation, formal analysis, methodology, and writing-original draft, review, and editing.

C Withers-Martinez: data curation and writing-original draft, review, and editing.

DA Baker: resources, supervision, funding acquisition, and writing-original draft, review, and editing.

MJ Blackman: conceptualization, resources, supervision, funding acquisition, and writing-original draft, review, and editing.

\section{Conflict of Interest Statement}

The authors declare that they have no conflict of interest.

\section{References}

1. Rothstein RJ (1983) One-step gene disruption in yeast. Methods Enzymol 101: 202-211. doi:10.1016/0076-6879(83)01015-0

2. Kilby NJ, Snaith MR, Murray JA (1993) Site-specific recombinases: Tools for genome engineering. Trends Genet 9: 413-421. doi:10.1016/01689525(93)90104-p

3. Cong L, Ran FA, Cox D, Lin S, Barretto R, Habib N, Hsu PD, Wu X, Jiang W, Marraffini LA, et al (2013) Multiplex genome engineering using CRISPR/ Cas systems. Science 339: 819-823. doi:10.1126/science.1231143

4. Jinek M, Chylinski K, Fonfara I, Hauer M, Doudna JA, Charpentier E (2012) A programmable dual-RNA-guided DNA endonuclease in adaptive bacterial immunity. Science 337: 816-821. doi:10.1126/science.1225829

5. World Health Organization (2018) World Malaria Report 2018. Geneva: World Health Organization. Licence: CC BY-NC-SA 3.0 IGO.

6. Burrows JN, Duparc S, Gutteridge WE, Hooft van Huijsduijnen R, Kaszubska W, Macintyre F, Mazzuri S, Mohrle JJ, Wells TNC (2017) New developments in anti-malarial target candidate and product profiles. Malar J 16: 26. doi:10.1186/s12936-017-1809-9

7. Goonewardene R, Daily J, Kaslow D, Sullivan TJ, Duffy P, Carter R, Mendis K, Wirth D (1993) Transfection of the malaria parasite and expression of firefly luciferase. Proc Natl Acad Sci U S A 90: 5234-5236. doi:10.1073/ pnas.90.11.5234

8. Sauer B, Henderson N (1988) Site-specific DNA recombination in mammalian cells by the Cre recombinase of bacteriophage P1. Proc Natl Acad Sci U S A 85: 5166-5170. doi:10.1073/pnas.85.14.5166

9. O'Neill MT, Phuong T, Healer J, Richard D, Cowman AF (2011) Gene deletion from Plasmodium falciparum using FLP and Cre recombinases: Implications for applied site-specific recombination. Int J Parasitol 41: 117-123. doi:10.1016/j.ijpara.2010.08.001

10. Collins CR, Das S, Wong EH, Andenmatten N, Stallmach R, Hackett F, Herman JP, Muller S, Meissner M, Blackman MJ (2013) Robust inducible Cre recombinase activity in the human malaria parasite Plasmodium falciparum enables efficient gene deletion within a single asexual erythrocytic growth cycle. Mol Microbiol 88: 687-701. doi:10.1111/mmi.12206

11. Andenmatten N, Egarter S, Jackson AJ, Jullien N, Herman JP, Meissner M (2013) Conditional genome engineering in Toxoplasma gondii uncovers alternative invasion mechanisms. Nat Methods 10: 125-127. doi:10.1038/ nmeth.2301

12. Siekierka JJ, Hung SH, Poe M, Lin CS, Sigal NH (1989) A cytosolic binding protein for the immunosuppressant FK506 has peptidyl-prolyl isomerase activity but is distinct from cyclophilin. Nature 341: 755-757. doi:10.1038/341755a0

13. Chiu MI, Katz H, Berlin V (1994) RAPT1, a mammalian homolog of yeast Tor, interacts with the FKBP12/rapamycin complex. Proc Natl Acad Sci U S A 91: 12574-12578. doi:10.1073/pnas.91.26.12574

14. Jullien N, Sampieri F, Enjalbert A, Herman JP (2003) Regulation of Cre recombinase by ligand-induced complementation of inactive fragments. Nucleic Acids Res 31: e131. doi:10.1093/nar/gng131

15. Jullien N, Goddard I, Selmi-Ruby S, Fina JL, Cremer H, Herman JP (2007) Conditional transgenesis using Dimerizable Cre (DiCre). PLoS One 2: e1355. doi:10.1371/journal.pone.0001355

16. Jones ML, Das S, Belda H, Collins CR, Blackman MJ, Treeck M (2016) A versatile strategy for rapid conditional genome engineering using loxP sites in a small synthetic intron in Plasmodium falciparum. Sci Rep 6: 21800. doi:10.1038/srep21800 
17. Yap A, Azevedo MF, Gilson PR, Weiss GE, O'Neill MT, Wilson DW, Crabb BS, Cowman AF (2014) Conditional expression of apical membrane antigen 1 in Plasmodium falciparum shows it is required for erythrocyte invasion by merozoites. Cell Microbiol 16: 642-656. doi:10.1111/cmi.12287

18. Birnbaum J, Flemming S, Reichard N, Soares AB, Mesen-Ramirez P, Jonscher E, Bergmann B, Spielmann T (2017) A genetic system to study Plasmodium falciparum protein function. Nat Methods 14: 450-456. doi:10.1038/nmeth.4223

19. Knuepfer E, Napiorkowska M, van Ooij C, Holder AA (2017) Generating conditional gene knockouts in Plasmodium: A toolkit to produce stable DiCre recombinase-expressing parasite lines using CRISPR/Cas9. Sci Rep 7: 3881. doi:10.1038/s41598-017-03984-3

20. Das S, Hertrich N, Perrin AJ, Withers-Martinez C, Collins CR, Jones ML, Watermeyer JM, Fobes ET, Martin SR, Saibil HR, et al (2015) Processing of Plasmodium falciparum merozoite surface protein MSP1 activates a spectrin-binding function enabling parasite egress from RBCs. Cell Host Microbe 18: 433-444. doi:10.1016/j.chom.2015.09.007

21. Collins CR, Hackett F, Atid J, Tan MSY, Blackman MJ (2017) The Plasmodium falciparum pseudoprotease SERA5 regulates the kinetics and efficiency of malaria parasite egress from host erythrocytes. PLOS Pathog 13: e1006453. doi:10.1371/journal.ppat.1006453

22. Pino P, Caldelari R, Mukherjee B, Vahokoski J, Klages N, Maco B, Collins CR, Blackman MJ, Kursula I, Heussler V, et al (2017) A multistage antimalarial targets the plasmepsins IX and $X$ essential for invasion and egress. Science 358: 522-528. doi:10.1126/science.aaf8675

23. Sherling ES, Knuepfer E, Brzostowski JA, Miller LH, Blackman MJ, van Ooij C (2017) The Plasmodium falciparum rhoptry protein RhopH3 plays essential roles in host cell invasion and nutrient uptake. Elife 6: e23239. doi:10.7554/elife.23239

24. Boonyalai N, Collins CR, Hackett F, Withers-Martinez C, Blackman MJ (2018) Essentiality of Plasmodium falciparum plasmepsin V. PLoS One 13: e0207621. doi:10.1371/journal.pone.0207621

25. Perrin AJ, Collins CR, Russell MRG, Collinson LM, Baker DA, Blackman MJ (2018) The actinomyosin motor drives malaria parasite red blood cell invasion but not egress. mBio 9: e00905-e00918. doi:10.1128/ mbio.00905-18

26. Thomas JA, Tan MSY, Bisson C, Borg A, Umrekar TR, Hackett F, Hale VL, Vizcay-Barrena G, Fleck RA, Snijders AP, et al (2018) A protease cascade regulates release of the human malaria parasite Plasmodium falciparum from host red blood cells. Nat Microbiol 3: 447-455. doi:10.1038/s41564-018-0111-0

27. Flueck C, Drought LG, Jones A, Patel A, Perrin AJ, Walker EM, Nofal SD, Snijders AP, Blackman MJ, Baker DA (2019) Phosphodiesterase beta is the master regulator of CAMP signalling during malaria parasite invasion. PLoS Biol 17: e3000154. doi:10.1371/journal.pbio.3000154

28. Patel A, Perrin AJ, Flynn HR, Bisson C, Withers-Martinez C, Treeck M, Flueck C, Nicastro G, Martin SR, Ramos A, et al (2019) Cyclic AMP signalling controls key components of malaria parasite host cell invasion machinery. PLoS Biol 17: e3000264. doi:10.1371/journal.pbio.3000264

29. Tiburcio M, Yang ASP, Yahata K, Suarez-Cortes P, Belda H, Baumgarten S, van de Vegte-Bolmer M, van Gemert GJ, van Waardenburg Y, Levashina EA, et al (2019) A novel tool for the generation of conditional knockouts to study gene function across the Plasmodium falciparum life cycle. mBio 10: e01170-19. doi:10.1128/mbio.01170-19

30. Kent RS, Modrzynska KK, Cameron R, Philip N, Billker O, Waters AP (2018) Inducible developmental reprogramming redefines commitment to sexual development in the malaria parasite Plasmodium berghei. Nat Microbiol 3: 1206-1213. doi:10.1038/s41564-018-0223-6

31. Knuepfer E, Wright KE, Kumar Prajapati S, Rawlinson TA, Mohring F, Koch M, Lyth OR, Howell SA, Villasis E, Snijders AP, et al (2019) Divergent roles for the RH5 complex components, CyRPA and RIPR in human-infective malaria parasites. PLoS Pathog 15: e1007809. doi:10.1371/journal. ppat.1007809
32. Deng W, Baker DA (2002) A novel cyclic GMP-dependent protein kinase is expressed in the ring stage of the Plasmodium falciparum life cycle. Mol Microbiol 44: 1141-1151. doi:10.1046/j.1365-2958.2002.02948.x

33. El Bakkouri M, Kouidmi I, Wernimont AK, Amani M, Hutchinson A, Loppnau P, Kim JJ, Flueck C, Walker JR, Seitova A, et al (2019) Structures of the CGMP-dependent protein kinase in malaria parasites reveal a unique structural relay mechanism for activation. Proc Natl Acad Sci U S A 116: 14164-14173. doi:10.1073/pnas.1905558116

34. McRobert L, Taylor CJ, Deng W, Fivelman QL, Cummings RM, Polley SD, Billker O, Baker DA (2008) Gametogenesis in malaria parasites is mediated by the CGMP-dependent protein kinase. PLoS Biol 6: e139. doi:10.1371/journal.pbio.0060139

35. Moon RW, Taylor CJ, Bex C, Schepers R, Goulding D, Janse CJ, Waters AP, Baker DA, Billker O (2009) A cyclic GMP signalling module that regulates gliding motility in a malaria parasite. PLoS Pathog 5: e1000599. doi:10.1371/journal.ppat.1000599

36. Falae A, Combe A, Amaladoss A, Carvalho T, Menard R, Bhanot P (2010) Role of Plasmodium berghei CGMP-dependent protein kinase in late liver stage development. J Biol Chem 285: 3282-3288. doi:10.1074/ jbc.m109.070367

37. Taylor HM, McRobert L, Grainger M, Sicard A, Dluzewski AR, Hopp CS, Holder AA, Baker DA (2010) The malaria parasite cyclic GMP-dependent protein kinase plays a central role in blood-stage schizogony. Eukaryot Cell 9: 37-45. doi:10.1128/ec.00186-09

38. Collins CR, Hackett F, Strath M, Penzo M, Withers-Martinez C, Baker DA Blackman MJ (2013) Malaria parasite CGMP-dependent protein kinase regulates blood stage merozoite secretory organelle discharge and egress. PLoS Pathog 9: e1003344. doi:10.1371/journal.ppat.1003344

39. Brochet M, Collins MO, Smith TK, Thompson E, Sebastian S, Volkmann K, Schwach F, Chappell L, Gomes AR, Berriman M, et al (2014) Phosphoinositide metabolism links CGMP-dependent protein kinase $G$ to essential $\mathrm{Ca}(2)(+)$ signals at key decision points in the life cycle of malaria parasites. PLoS Biol 12: e1001806. doi:10.1371/journal. pbio.1001806

40. Govindasamy K, Jebiwott S, Jaijyan DK, Davidow A, Ojo KK, Van Voorhis WC, Brochet M, Billker O, Bhanot P (2016) Invasion of hepatocytes by Plasmodium sporozoites requires CGMP-dependent protein kinase and calcium dependent protein kinase 4. Mol Microbiol 102: 349-363. doi:10.1111/mmi.13466

41. Baker DA, Stewart LB, Large JM, Bowyer PW, Ansell KH, Jimenez-Diaz MB, El Bakkouri M, Birchall K, Dechering KJ, Bouloc NS, et al (2017) A potent series targeting the malarial CGMP-dependent protein kinase clears infection and blocks transmission. Nat Commun 8: 430. doi:10.1038/ s41467-017-00572-X

42. Ganter M, Goldberg JM, Dvorin JD, Paulo JA, King JG, Tripathi AK, Paul AS, Yang J, Coppens I, Jiang RH, et al (2017) Plasmodium falciparum CRK4 directs continuous rounds of DNA replication during schizogony. Nat Microbiol 2: 17017. doi:10.1038/nmicrobiol.2017.17

43. Bishop AC, Ubersax JA, Petsch DT, Matheos DP, Gray NS, Blethrow J, Shimizu E, Tsien JZ, Schultz PG, Rose MD, et al (2000) A chemical switch for inhibitor-sensitive alleles of any protein kinase. Nature 407: 395-401. doi:10.1038/35030148

44. Deakin A, Duddy G, Wilson S, Harrison S, Latcham J, Fulleylove M, Fung S, Smith J, Pedrick M, McKevitt T, et al (2014) Characterisation of a K390R ITK kinase dead transgenic mouse: Implications for ITK as a therapeutic target. PLoS One 9: e107490. doi:10.1371/journal.pone.0107490

45. Patrucco E, Notte A, Barberis L, Selvetella G, Maffei A, Brancaccio M, Marengo S, Russo G, Azzolino O, Rybalkin SD, et al (2004) PI3Kgamma modulates the cardiac response to chronic pressure overload by distinct kinase-dependent and -independent effects. Cell 118: 375-387. doi:10.1016/j.cell.2004.07.017

46. Ventura S, Cano F, Kannan Y, Breyer F, Pattison MJ, Wilson MS, Ley SC (2018) A20-binding inhibitor of NF-kappaB (ABIN) 2 negatively regulates 
allergic airway inflammation. J Exp Med 215: 2737-2747. doi:10.1084/ jem.20170852

47. Weiss EL, Bishop AC, Shokat KM, Drubin DG (2000) Chemical genetic analysis of the budding-yeast p21-activated kinase Cla4p. Nat Cell Biol 2: 677-685. doi:10.1038/35036300

48. Arencibia JM, Pastor-Flores D, Bauer AF, Schulze JO, Biondi RM (2013) AGC protein kinases: From structural mechanism of regulation to allosteric drug development for the treatment of human diseases. Biochim Biophys Acta 1834: 1302-1321. doi:10.1016/j.bbapap. 2013.03.010

49. Treeck M, Sanders JL, Elias JE, Boothroyd JC (2011) The phosphoproteomes of Plasmodium falciparum and Toxoplasma gondii reveal unusual adaptations within and beyond the parasites' boundaries. Cell Host Microbe 10: 410-419. doi:10.1016/j.chom. 2011.09.004

50. Lasonder E, Green JL, Camarda G, Talabani H, Holder AA, Langsley G, Alano P (2012) The Plasmodium falciparum schizont phosphoproteome reveals extensive phosphatidylinositol and CAMP-protein kinase A signaling. J Proteome Res 11: 5323-5337. doi:10.1021/pr300557m

51. Pease BN, Huttlin EL, Jedrychowski MP, Talevich E, Harmon J, Dillman T, Kannan N, Doerig C, Chakrabarti R, Gygi SP, et al (2013) Global analysis of protein expression and phosphorylation of three stages of Plasmodium falciparum intraerythrocytic development. J Proteome Res 12: 4028-4045. doi:10.1021/pr400394g

52. Collins MO, Wright JC, Jones M, Rayner JC, Choudhary JS (2014) Confident and sensitive phosphoproteomics using combinations of collision induced dissociation and electron transfer dissociation. J Proteomics 103: 1-14. doi:10.1016/j.jprot.2014.03.010

53. Lasonder E, Green JL, Grainger M, Langsley G, Holder AA (2015) Extensive differential protein phosphorylation as intraerythrocytic Plasmodium falciparum schizonts develop into extracellular invasive merozoites. Proteomics 15: 2716-2729. doi:10.1002/pmic.201400508

54. Livet J, Weissman TA, Kang H, Draft RW, Lu J, Bennis RA, Sanes JR, Lichtman JW (2007) Transgenic strategies for combinatorial expression of fluorescent proteins in the nervous system. Nature 450: 56-62. doi:10.1038/nature06293

55. Lee G, Saito I (1998) Role of nucleotide sequences of loxP spacer region in Cre-mediated recombination. Gene 216: 55-65. doi:10.1016/S0378 1119(98)00325-4

56. Kooij TW, Rauch MM, Matuschewski K (2012) Expansion of experimental genetics approaches for Plasmodium berghei with versatile transfection vectors. Mol Biochem Parasitol 185: 19-26. doi:10.1016/ j.molbiopara.2012.06.001

57. Hopp CS, Flueck C, Solyakov L, Tobin A, Baker DA (2012) Spatiotemporal and functional characterisation of the Plasmodium falciparum CGMPdependent protein kinase. PLoS One 7: e48206. doi:10.1371/ journal.pone.0048206

58. Glushakova S, Beck JR, Garten M, Busse BL, Nasamu AS, Tenkova-Heuser T, Heuser J, Goldberg DE, Zimmerberg J (2018) Rounding precedes rupture and breakdown of vacuolar membranes minutes before malaria parasite egress from erythrocytes. Cell Microbiol 20: e12868. doi:10.1111/ cmi.12868

59. Glushakova S, Yin D, Li T, Zimmerberg J (2005) Membrane transformation during malaria parasite release from human red blood cells. Curr Biol 15: 1645-1650. doi:10.1016/j.cub.2005.07.067

60. Hale VL, Watermeyer JM, Hackett F, Vizcay-Barrena G, van Ooij C, Thomas JA, Spink MC, Harkiolaki M, Duke E, Fleck RA, et al (2017) Parasitophorous vacuole poration precedes its rupture and rapid host erythrocyte cytoskeleton collapse in Plasmodium falciparum egress. Proc Natl Acad Sci U S A 114: 3439-3444. doi:10.1073/pnas.1619441114

61. Zheng B, Sage M, Sheppeard EA, Jurecic V, Bradley A (2000) Engineering mouse chromosomes with Cre-loxP: Range, efficiency, and somatic applications. Mol Cell Biol 20: 648-655. doi:10.1128/mcb.20.2.648 655.2000

62. Delplace P, Bhatia A, Cagnard M, Camus D, Colombet G, Debrabant A, Dubremetz JF, Dubreuil N, Prensier G, Fortier B, et al (1988) Protein p126: A parasitophorous vacuole antigen associated with the release of Plasmodium falciparum merozoites. Biol Cell 64: 215-221. doi:10.1016/ 0248-4900(88)90080-9

63. Albert H, Dale EC, Lee E, Ow DW (1995) Site-specific integration of DNA into wild-type and mutant lox sites placed in the plant genome. Plant 17 : 649-659. doi:10.1046/j.1365-313x.1995.7040649.x

64. Hampel S, Chung P, McKellar CE, Hall D, Looger LL, Simpson JH (2011) Drosophila Brainbow: A recombinase-based fluorescence labeling technique to subdivide neural expression patterns. Nat Methods 8: 253-259. doi:10.1038/nmeth.1566

65. Pan YA, Freundlich T, Weissman TA, Schoppik D, Wang XC, Zimmerman S, Ciruna B, Sanes JR, Lichtman JW, Schier AF (2013) Zebrabow: Multispectral cell labeling for cell tracing and lineage analysis in zebrafish. Development 140: 2835-2846. doi:10.1242/dev.094631

66. Garcia-Moreno F, Vasistha NA, Begbie J, Molnar Z (2014) CLoNe is a new method to target single progenitors and study their progeny in mouse and chick. Development 141: 1589-1598. doi:10.1242/dev.105254

67. Mesen-Ramirez P, Bergmann B, Tran TT, Garten M, Stacker J, NaranjoPrado I, Hohn K, Zimmerberg J, Spielmann T (2019) EXP1 is critical for nutrient uptake across the parasitophorous vacuole membrane of malaria parasites. PLoS Biol 17: e3000473. doi:10.1371/journal. pbio.3000473

68. O'Donnell RA, Freitas-Junior LH, Preiser PR, Williamson DH, Duraisingh M McElwain TF, Scherf A, Cowman AF, Crabb BS (2002) A genetic screen for improved plasmid segregation reveals a role for Rep20 in the interaction of Plasmodium falciparum chromosomes. EMBO J 21: 1231-1239. doi:10.1093/emboj/21.5.1231

69. O'Donnell RA, Preiser PR, Williamson DH, Moore PW, Cowman AF, Crabb BS (2001) An alteration in concatameric structure is associated with efficient segregation of plasmids in transfected Plasmodium falciparum parasites. Nucleic Acids Res 29: 716-724. doi:10.1093/nar/29.3.716

70. Andreadaki M, Deligianni E, Nika F, Siden-Kiamos I (2016) Expression of the Plasmodium berghei actin II gene is controlled by elements in a long genomic region. Parasitol Res 115: 3261-3265. doi:10.1007/ s00436-016-5133-z

71. Bansal A, Molina-Cruz A, Brzostowski J, Liu P, Luo Y, Gunalan K, Li Y, Ribeiro JMC, Miller LH (2018) PfCDPK1 is critical for malaria parasite gametogenesis and mosquito infection. Proc Natl Acad Sci U S A 115: 774-779. doi:10.1073/pnas.1715443115

72. Lehmann C, Tan MSY, de Vries LE, Russo I, Sanchez MI, Goldberg DE, Deu E (2018) Plasmodium falciparum dipeptidyl aminopeptidase 3 activity is important for efficient erythrocyte invasion by the malaria parasite. PLoS Pathog 14: e1007031. doi:10.1371/journal.ppat.1007031

73. Goldberg DE, Janse CJ, Cowman AF, Waters AP (2011) Has the time come for us to complement our malaria parasites? Trends Parasitol 27: 1-2. doi:10.1016/j.pt.2010.06.017

74. Pontes-Quero S, Heredia L, Casquero-Garcia V, Fernandez-Chacon M, Luo W, Hermoso A, Bansal M, Garcia-Gonzalez I, Sanchez-Munoz MS, Perea JR, et al (2017) Dual ifgMosaic: A versatile method for multispectral and combinatorial mosaic gene-function analysis. Cell 170: 800-814.e18. doi:10.1016/j.cell.2017.07.031

75. Ganesan SM, Falla A, Goldfless SJ, Nasamu AS, Niles JC (2016) Synthetic RNA-protein modules integrated with native translation mechanisms to control gene expression in malaria parasites. Nat Commun 7: 10727 doi:10.1038/ncomms10727

76. Prommana $\mathrm{P}$, Uthaipibull C, Wongsombat C, Kamchonwongpaisan S, Yuthavong Y, Knuepfer E, Holder AA, Shaw PJ (2013) Inducible knockdown of Plasmodium gene expression using the glmS ribozyme. PLoS One 8 : e73783. doi:10.1371/journal.pone.0073783 
77. Armstrong CM, Goldberg DE (2007) An FKBP destabilization domain modulates protein levels in Plasmodium falciparum. Nat Methods 4: 1007-1009. doi:10.1038/nmeth1132

78. Goldfless SJ, Wagner JC, Niles JC (2014) Versatile control of Plasmodium falciparum gene expression with an inducible protein-RNA interaction. Nat Commun 5: 5329. doi:10.1038/ncomms6329

79. Hughes KR, Waters AP (2017) Rapid inducible protein displacement in Plasmodiumin vivo and in vitro using knocksideways technology. Wellcome Open Res 2: 18. doi:10.12688/wellcomeopenres.11005.1

80. Stallmach R, Kavishwar M, Withers-Martinez C, Hackett F, Collins CR, Howell SA, Yeoh S, Knuepfer E, Atid AJ, Holder AA, et al (2015) Plasmodium falciparum SERA5 plays a non-enzymatic role in the malarial asexual blood-stage lifecycle. Mol Microbiol 96: 368-387. doi:10.1111/mmi.12941

81. Moon RW, Hall J, Rangkuti F, Ho YS, Almond N, Mitchell GH, Pain A, Holder AA, Blackman MJ (2013) Adaptation of the genetically tractable malaria pathogen Plasmodium knowlesi to continuous culture in human erythrocytes. Proc Natl Acad Sci U S A 110: 531-536. doi:10.1073/ pnas. 1216457110

82. Thomas JA, Collins CR, Das S, Hackett F, Graindorge A, Bell D, Deu E, Blackman MJ (2016) Development and application of a simple plaque assay for the human malaria parasite Plasmodium falciparum. PLoS One 11: e0157873. doi:10.1371/journal. pone.0157873

83. Schindelin J, Arganda-Carreras I, Frise E, Kaynig V, Longair M, Pietzsch T, Preibisch S, Rueden C, Saalfeld S, Schmid B, et al (2012) Fiji: An opensource platform for biological-image analysis. Nat Methods 9: 676-682. doi:10.1038/nmeth.2019

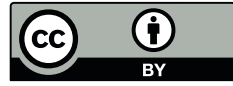

License: This article is available under a Creative Commons License (Attribution 4.0 International, as described at https://creativecommons.org/ licenses/by/4.0/). 